\title{
Signaling in the immune response ${ }^{*}$
}

\author{
Jonathan J. Ewbank ${ }^{\S}$, Centre d'Immunologie de Marseille-Luminy, \\ INSERM/CNRS/Université de la Méditerranée, 13288 Marseille cedex 9 , \\ France
}

\section{Table of Contents}

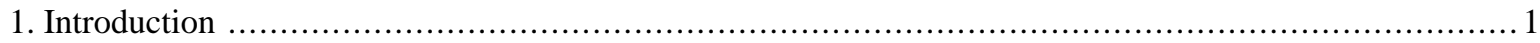

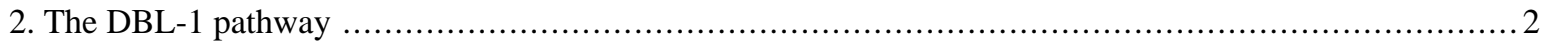

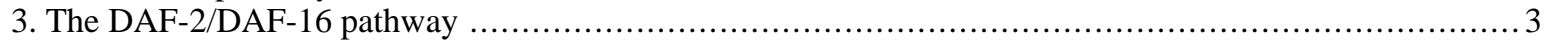

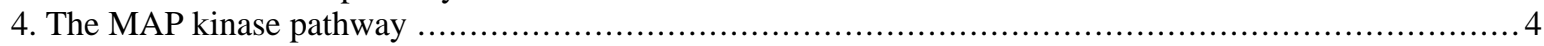

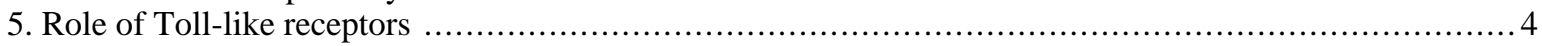

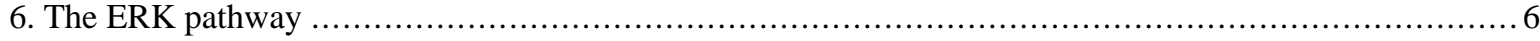

7. Recognition of infection through stress response ....................................................... 7

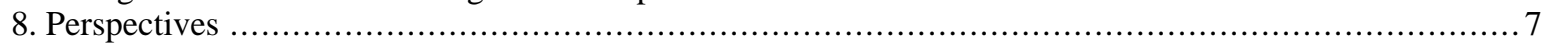

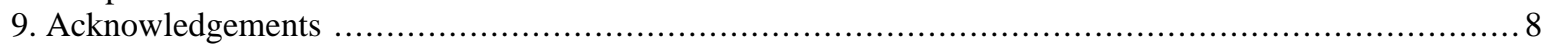

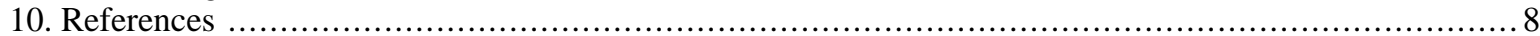

\begin{abstract}
Many pathogens that can infect $C$. elegans have been described, including some that co-exist with the nematode in its natural environment. This chapter describes our current understanding of the different innate immune responses of $C$. elegans that follow infection. It focuses on the main signalling pathways that have been identified and highlights the inclusion of certain molecular cassettes in both immune and developmental functions.
\end{abstract}

\section{Introduction}

C. elegans has emerged as an extremely powerful model for the study of innate immunity, that is to say the fundamental aspects of a host's response to infection, complementing more established models such as Drosophila (Kurz and Ewbank, 2003). In 1999, the Ausubel group published work showing that $C$. elegans could be infected by Pseudomonas aeruginosa, a ubiquitous Gram negative bacterium associated with chronic lung infections in cystic fibrosis patients, and that specific worm mutants had an increased susceptibility to lethal intoxication (Mahajan-Miklos et al., 1999). Work from the Ausubel group also showed that worms can be used to identify universal virulence factors that are required for bacterial pathogenesis regardless of the host (reviewed in Mahajan-Miklos et al., 2000; Tan et al., 1999a; Tan et al., 1999b). In the years since these publications, many

\footnotetext{
*Edited by Iva Greenwald. Last revised February 7, 2005. Published January 23, 2006. This chapter should be cited as: Ewbank, J. J. Signaling in the immune response (January 23, 2006), WormBook, ed. The C. elegans Research Community, WormBook, doi/10.1895/wormbook.1.83.1, http://www.wormbook.org.

Copyright: (C) 2006 Jonathan J. Ewbank. This is an open-access article distributed under the terms of the Creative Commons Attribution License, which permits unrestricted use, distribution, and reproduction in any medium, provided the original author and source are credited

${ }^{\S}$ To whom correspondence should be addressed. E-mail: ewbank@ciml.univ-mrs.fr
} 
different microbes that can infect worms have been identified and there has been a wealth of studies using $C$. elegans to investigate bacterial pathogenesis as well as innate immunity. These have helped define the relevance of the model for understanding host-pathogen interactions in higher organisms (reviewed in Alegado et al., 2003; Kurz and Ewbank, 2003; Kurz and Tan, 2004; Millet and Ewbank, 2004).

This chapter will deal with the pathways involved in the response of $C$. elegans to infection. A recurrent theme will be that the signaling pathways involved in innate defenses involve protein cassettes that play essential roles in other, seemingly unrelated developmental or physiological processes.

The current view of worm defenses has been obtained through a variety of methods, including forward genetic screens, RNAi, microarrays and candidate gene approaches. At least 4 pathways have been shown to be involved in innate immunity in $C$. elegans and an overlap has been found between the genes involved in pathogen defense and longevity. In hindsight, this is not so surprising since the standard E. coli strain OP50 is not entirely benign. Under certain culture conditions it is capable of intoxicating worms rapidly (Garsin et al., 2001) and even on standard NGM plates, colonization of the intestine by OP50, with resultant tissue damage, appears to be one of the principal reasons why old worms die (Gems and Riddle, 2000; Herndon et al., 2002; reviewed in Alegado et al., 2003; Kurz and Tan, 2004).

Infection of $C$. elegans causes specific changes in gene regulation. In the case of bacterial pathogens that colonize the gut, the induced genes include those encoding antimicrobial peptides and proteins, (e.g., certain caenacins and lysozymes, respectively). Many are specifically expressed in the intestinal cells and there is circumstantial evidence to suggest that the peptides and proteins are secreted into the gut lumen and directly target any microorganisms that are there, thereby contributing to the worms' defenses (Kurz and Tan, 2004; Mallo et al., 2002). They may act in synergy with constitutively expressed antimicrobial proteins and peptides, including the ABF (anti-bacterial factor) family (Kato et al., 2002).

\section{The DBL-1 pathway}

The resistance of worms to infection by $P$. aeruginosa and a second human opportunistic Gram-negative bacterial pathogen, Serratia marcescens, involves in part the DBL-1 pathway (Mallo et al., 2002; Tan, 2001). The gene $d b l-1$ (for Dpp, BMP-like; previously referred to as $c e t-1$ ) encodes one of 4 TGF- $\beta$-like ligands in the worm. DBL-1 binds the heterodimeric DAF-4/SMA-6 receptor and acts via the SMA-2/SMA-3/SMA-4 SMAD complex to control gene expression (Figure 1). It has a role in determining the structure of the male tail, acting through mab-21 (Morita et al., 1999), and in controlling body size and hypodermal and intestinal cell polyploidy via lon-1 (Maduzia et al., 2002; Morita et al., 2002). Based on its similarity to the plant defense protein PR-1, we previously speculated on a direct antimicrobial role for LON-1 (Kurz and Ewbank, 2003). But in fact, neither mab-21 nor lon-1 are required for resistance to $P$. aeruginosa (M.-W. Tan, personal communication), while sma-2, $-3,-4$ and -6 are. Thus loss of function mutants in the latter four genes are hypersensitive to infection (Mallo et al., 2002; Tan, 2001). It therefore appears that the upstream part of a single signaling pathway is used in very distinct contexts and can target different genes when triggered by different stimuli. How multiple independent genes can be specifically regulated as a function of the activation of one pathway is only just beginning to be understood even in simpler model systems (Seet and Pawson, 2004). In part, the explanation likely comes from pathway cross-talk, the interdependence of signal transduction mechanisms (see, for example, Millet and Ewbank, 2004) and the temporal and spatial restriction of co-factor expression. As an example of the latter mechanism, it is known that the SMA-2/SMA-3/SMA-4 complex can act in association with certain of the multiple isoforms of SMA-9, a zinc finger transcription factor, and that the different sma-9 transcripts do have different patterns of expression (Liang et al., 2003). 


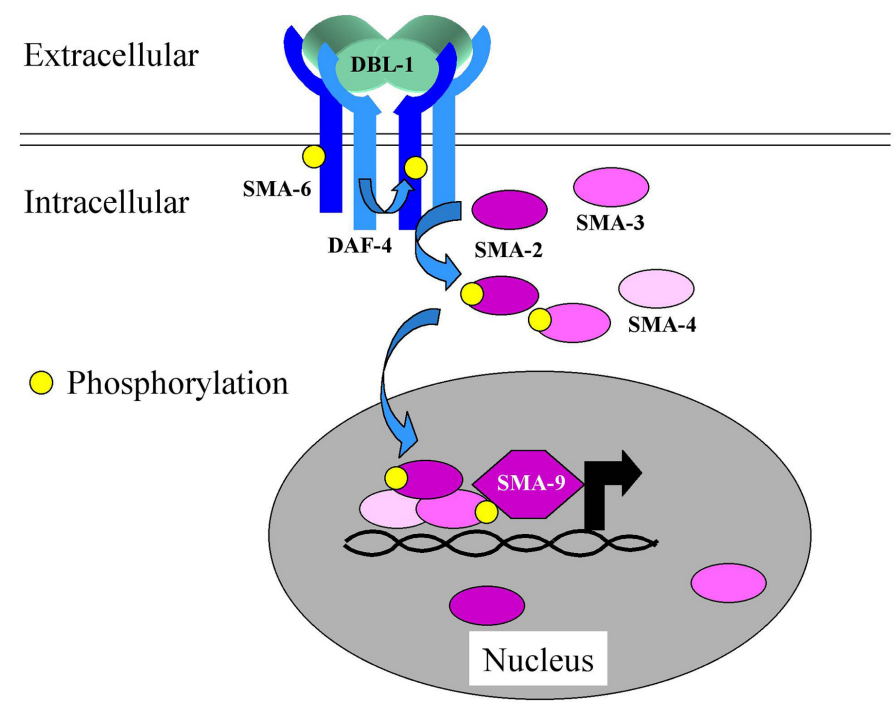

Figure 1. The DBL-1/TGF- $\beta$ pathway. Binding of the TGF- $\beta$-like ligand to the heterodimeric DAF-4/SMA-6 receptor leads to the phosphorylation and activation of the SMAD proteins SMA-2, SMA-3 and SMA-4. They translocate into the nucleus where they can activate gene expression, in some cases in association with SMA-9.

\section{The DAF-2/DAF-16 pathway}

One of the $d b l-1$ pathway targets that is upregulated by bacterial infection is the lysozyme gene lys- 8 . A second pathway shown to be involved in antibacterial defenses, the DAF-2/DAF-16 pathway (Figure 2), also regulates its expression (Murphy et al., 2003). Alluded to above, this pathway has been well characterized for its role in the control of longevity in C. elegans.

(A)
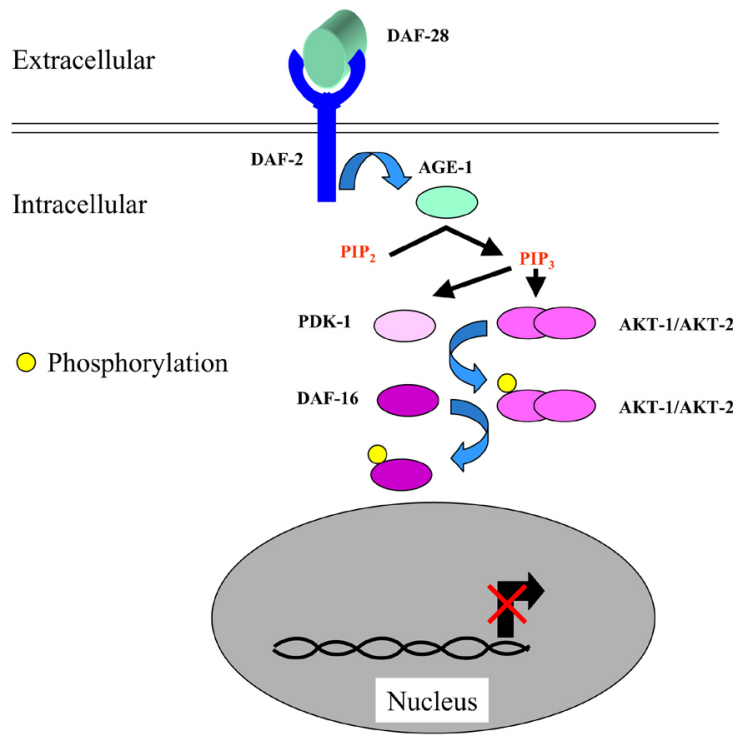

(B)
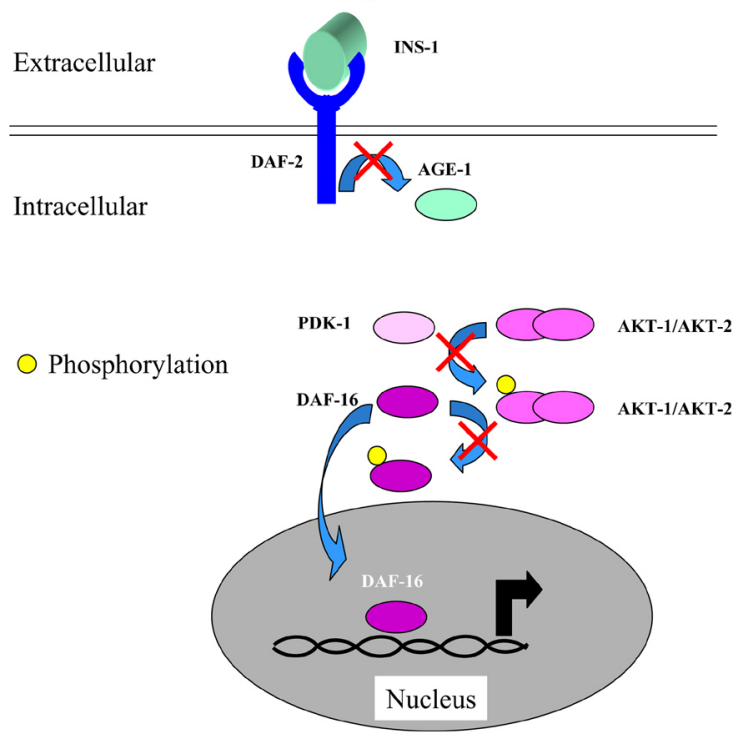

Figure 2. The DAF-2/DAF-16 pathway. (A) In the presence of an agonist ligand, such as the insulin-like peptide DAF-28, the DAF-2 receptor is activated and in turn activates the phosphatidylinositol-3 OH kinase AGE-1 that catalyses the conversion of phosphatidylinositol bisphosphate (PIP) into phosphatidylinositol trisphosphate (PIP ).On one hand, PIP binds to the complex AKT-1/AKT-2 and leads to the exposure of two phosphorylation sites. On the other hand, the kinase PDK-1 by binding to PiP is recruited to the membrane where it can phosphorylate and activate AKT- 1 . The kinase AKT in turn phosphorylates the transcription factor DAF-16 and thereby ensure its cytoplasmic retention. (B) In the presence of an antagonist ligand such as INS-1, (or in a daf-2 loss of function mutant), the pathway is not active, DAF-16 is not phosphorylated and can be translocated to the nucleus where it regulates the expression of a set of stress response and antimicrobial genes.

Not only are daf-2 mutants long-lived on a diet of OP50, but they also resist infection by a range of both Gram-negative and Gram-positive bacterial pathogens (Garsin et al., 2003). Known targets of the DAF-2/DAF-16 
pathway include the poorly characterized members of the $d o d$ (downstream of $\underline{D} A F-16$ ) gene group as well as patent antimicrobial genes, such as lys-7, those encoding saposins (spp-1 and spp-12, two of the worm's 20 spp genes) and the nematode thaumatin homologues (that in plants are antifungal proteins; Murphy et al., 2003). The pathway is assumed to contribute to resistance to infection by regulating the production of these different secreted antimicrobial proteins, as described for the DBL-1 pathway above. Indeed, abrogation of the expression of several of these proteins (e.g., SPP-1, SPP-12 and LYS-7) in daf-2 mutants leads to reduction in lifespan (Murphy et al., 2003). It is noteworthy that the expression of LYS-1 and LYS-2, SPP-3 and SPP-18 is also strongly upregulated upon $P$. aeruginosa infection (Kurz and Tan, 2004).

\section{The MAP kinase pathway}

Resistance to $P$. aeruginosa has been shown to involve a third pathway, a MAP kinase pathway, involving the MAP3K NSY-1 and the MAP2K SEK-1 that had originally been characterized as playing a role in determining asymmetric neuronal cell fate. While in wild-type worms the chemoreceptor STR-2 is only expressed in one of the two AWC neurons, in nsy-1 and sek-1 mutants, both sister neurons express the receptor (Sagasti et al., 2001; Tanaka-Hino et al., 2002). Alleles of $n s y-1$ and sek- 1 were found in a genetic screen for worms hyper-susceptible to $P$. aeruginosa and the two genes were shown to act together upstream of pmk-1. RNAi against this gene, which encodes one of the worm's three p38-family MAP kinases, also results in an increased susceptibility to infection (Kim et al., 2002). While in the context of cell determination $n s y-1$ and sek- 1 act downstream of unc- 43 that encodes a calcium-calmodulin-dependent kinase, this latter gene has been reported not to play a role in innate immune signaling (Kim et al., 2002). Further, abrogation of pmk-1 function by RNAi does not provoke a cell determination phenotype, suggesting that the downstream components of the pathway may also differ in the two contexts (Chuang and Bargmann, 2005). On the other hand, as discussed below, the adaptor protein TIR-1 was shown to be involved in both neuronal and defense pathways (Figure 3).

\section{CALCIUM SIGNALLING}
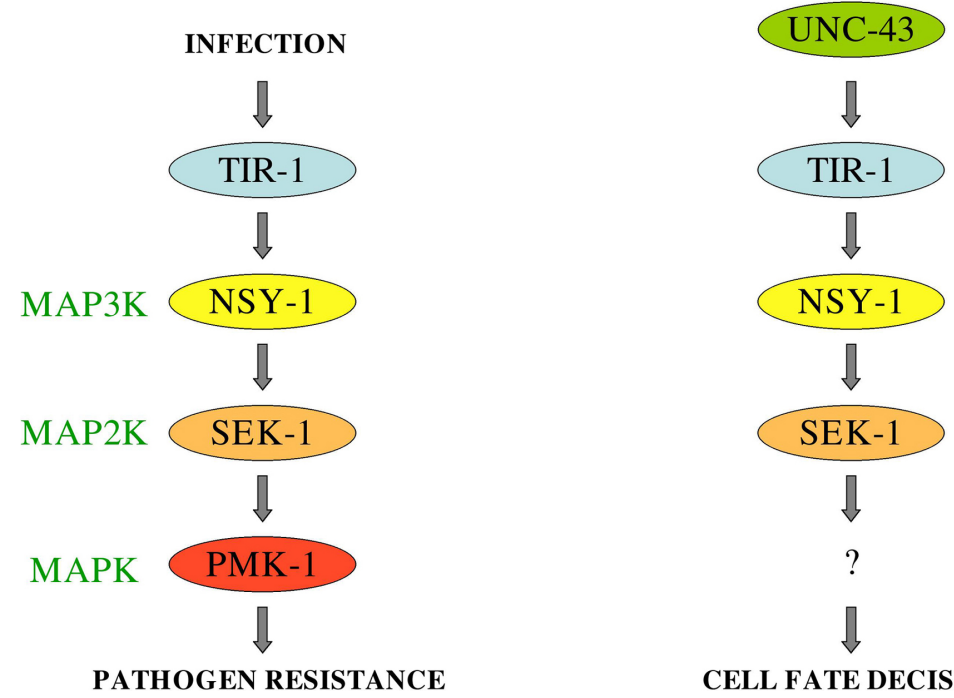

CELL FATE DECISION

Figure 3. The TIR-1/NSY-1/SEK-1 cassette functions in innate immunity and cell fate determination. See text for details.

\section{Role of Toll-like receptors}

P. aeruginosa and S. marcescens are both extracellular pathogens with a broad host range. Salmonella typhimurium on the other hand causes very specific diseases in mammals, associated with its entry into, and subsequent replication within host cells. Many different Salmonella strains can infect worms, but they do not behave as intracellular pathogens, and instead establish a permanent and debilitating colonization of the intestine (Aballay et al., 2000; Labrousse et al., 2000). Infection of C. elegans provokes germline apoptosis in a pmk 1-dependent fashion (Aballay and Ausubel, 2001; Aballay et al., 2003). Salmonella mutants with an altered lipopolysaccharide (LPS; one of the main components of the Gram-negative bacterial outer membrane) do not trigger the response. In vertebrates, the Toll-like receptor TLR4 functions in LPS-recognition, but the sole worm TLR, encoded by tol-1, is not necessary for Salmonella-induced apoptosis (Aballay et al., 2003). 
In Drosophila, activation of Toll leads to the expression of antifungal peptides. It appears that tol- 1 is not directly involved in regulating the expression of antimicrobial peptides, nor does it contribute to worms' resistance to fungal infection (Couillault et al., 2004), although it does play an enigmatic role in a specific behavioral response that leads N2 worms to avoid pathogenic S. marcescens, and is essential for embryonic development (Pujol and Ewbank, 2003; Pujol et al., 2001). Activation of TLRs in flies and mammals, where they play key roles in innate immunity, leads to the nuclear import of NF- $\mathrm{KB}$ family transcription factors. There is no evident NF- $\mathrm{KB}$ homolog in C. elegans. There are several interpretations of these findings. Assuming a phylogeny that places C. elegans and Drosophila in the same clade (the Ecdysozoa), we previously proposed that the worm lost NF- $\mathrm{KB}$ and the associated signaling pathways (Kurz and Ewbank, 2003). But, if flies are closer to humans in evolutionary terms than worms are, a role for TLRs in defense may have evolved following the acquisition of NF- $\kappa B$, after the split of the nematodes from a common fly-human ancestor. As an alternative, it has been suggested that a role for TLRs in innate immune signaling arose independently in protostomial and deuterostomial coelomates, possibly for controlling microbial infection in the body cavity (Kanzok et al., 2004). It is noteworthy that work from H. Fares has shown that $C$. elegans is extremely susceptible to the intra-pseudocoelomic injection of even small numbers of non-pathogenic bacteria, and that the coelomocytes appear not to have the capacity to phagocytose foreign particles (reported in Ewbank, 2002). Currently, nothing is known of the signaling pathway that is downstream of TOL-1, but it would be interesting to characterize as it could represent the earliest context for TLR function.

TLRs share a common structure, and are comprised of an ectodomain of leucine-rich repeats and an intracellular TIR (for Toll and Interleukin-1 receptor) domain that is also present in a number of plant disease resistance proteins. In mammals, the functionally characterized TLRs all act via TIR-domain containing adaptor proteins that serve as signal transducers ultimately provoking a pro-inflammatory response via NF- $\kappa \mathrm{B}$. There are 5 members of the TIR-domain adaptor family, and 4 of them, MyD88, TIRAP/Mal, TICAM/Trif and TRAM have known roles in TLR signal transduction. The fifth, SARM has no known function, but is the only one to have a direct orthologue in C. elegans. Given the importance of TIR-domain proteins in both plants and animals, it was natural that the role of tir- 1 in nematode defences was evaluated. It turned out that the gene is important for resistance to the bacterial pathogens $S$. marcescens and $P$. aeruginosa but not apparently for normal longevity (Couillault et al., 2004; Liberati et al., 2004). RNAi directed against all 5 tir-1 isoforms provokes an increased susceptibility not only to bacterial infection but also to the fungus Drechmeria coniospora. Indeed, tir-1 was shown to be necessary for the induction of antimicrobial peptide expression provoked by infection (Couillault et al., 2004), specifically that of NLP-29 and NLP-31 (Figure 4). The expression, and presumably secretion, of these glycine- and tyrosine-rich peptides is increased following infection by D. coniospora. Significantly, tir-1 appears to act in a TLR-independent fashion, suggesting that it may participate in a hitherto uncharacterised innate defence pathway (Couillault et al., 2004). On the other hand, as mentioned above, it acts together with $n s y-1$ and sek- 1 (Liberati et al., 2004), and in fact was identified (under the name of nsy-2) in the original screen for genes necessary for the asymmetric expression of str-2 in the AWC pair of neurons. Further, it has been shown that TIR-1, NSY-1 and SEK-1 can be co-immunoprecipitated when co-expressed in cell culture, and so probably form a physical complex (Chuang and Bargmann, 2005). These results, and the fact that PMK-1 phosphorylation is tir-1-dependent (Liberati et al., 2004), thus place the gene firmly in a MAPK pathway, but suggest that its activation in the context of infection in adult worms is different from that seen in the AWC neurons during development (Figure 3). Significantly, the enhanced susceptibility to $P$. aeruginosa infection of sek-1 mutants can be rescued by intestine-specific expression of a wild-type transgene (Sakaguchi et al., 2004). tir- 1 has also been shown to interact in two-hybrid screens with RAB-1 and the f subunit of ATP synthase. As RNAi of the corresponding genes phenocopies tir-1 RNAi, these interactions would seem to be real (Couillault et al., 2004). For the moment their functional integration with tir- 1 in the cell remains obscure. Additionally, the putative receptor involved in pathogen recognition upstream of tir- 1 has yet to be identified Genetic screens are currently underway to try to fill these lacunae (C. L. Kurz and N. Pujol, unpublished results). 

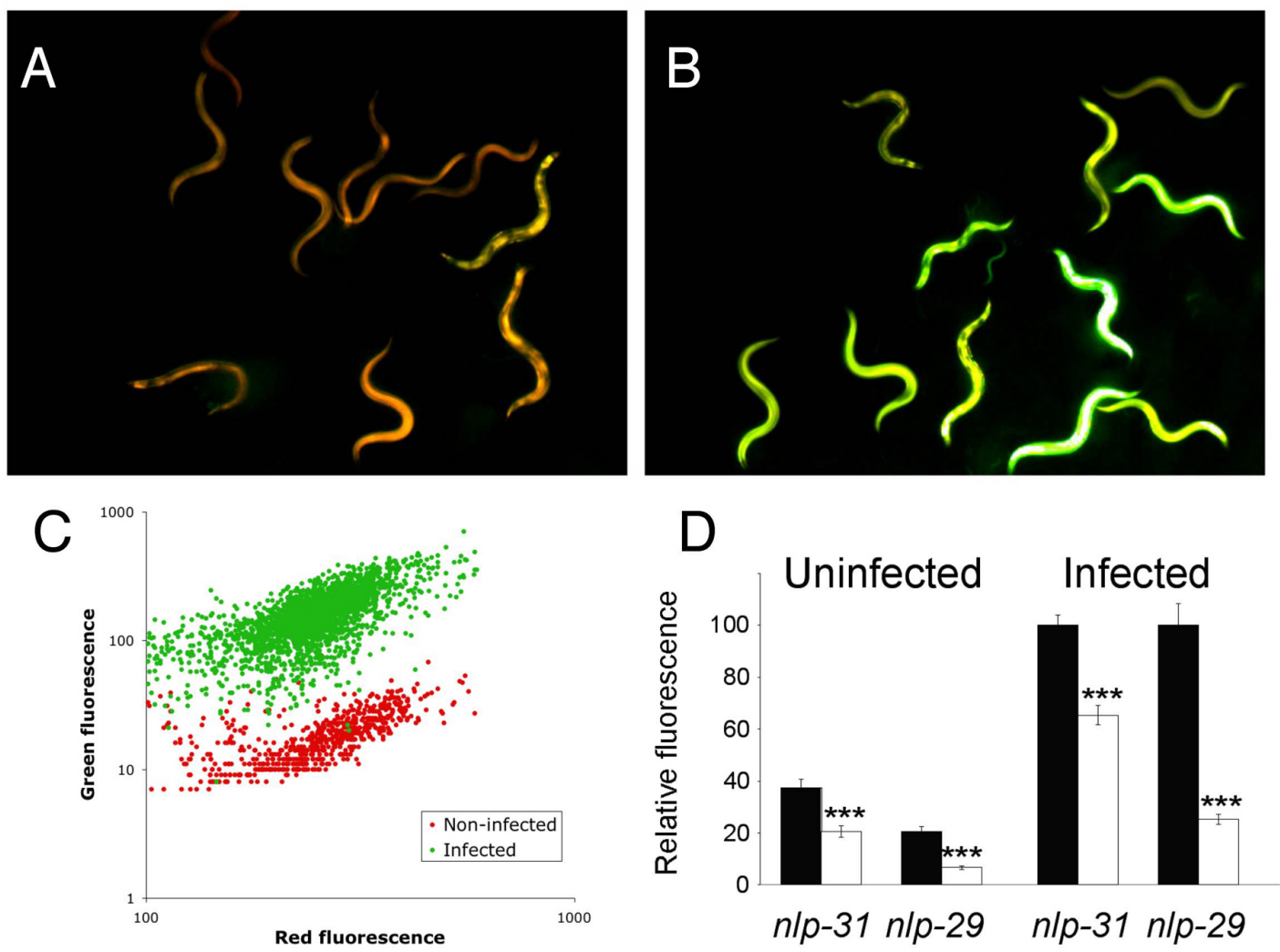

Figure 4. Control of antimicrobial peptide expression by tir-1. (A) Transgenic worms carrying a pnlp 29::gfp and a pcol-12::dsRed reporter constructs appear predominantly red in the absence of infection due to the constitutive activity of the col-12 hypodermis-specific promoter. (B) Following infection by the fungus Drechmeria coniospora the pnlp 29::gfp reporter is induced and the worms appear yellowy-green. (C) The relative level of induction varies between worms as quantified with the Union Biometrica COPAS biosort. Red and green fluorescence is quantified in arbitrary but constant units. (D) Quantification of the induction of pnlp $29: \because g f p$ and pnlp $31: \because g f p$ reporter gene expression following D. coniospora infection and the effect of control RNAi (black bars) or RNAi against all tir- 1 isoforms (white bars). Images in A and B provided by Nathalie Pujol; data in C generated by Aurélie Blanc; D adapted from Couillault et al. (2004).

\section{The ERK pathway}

A different MAPK pathway, the ERK pathway involving LIN-45 and MEK-2 and MPK-1 (Figure 5), is involved in the response of worms to the Gram-positive bacterial pathogen Microbacterium nematophilum (Nicholas and Hodgkin, 2004). In this case, the bacteria adhere to a specific rectal and peri-anal region of the cuticle and induce a conspicuous swelling (Hodgkin et al., 2000) that constitutes a specific host defense mechanism (Gravato-Nobre et al., 2005).

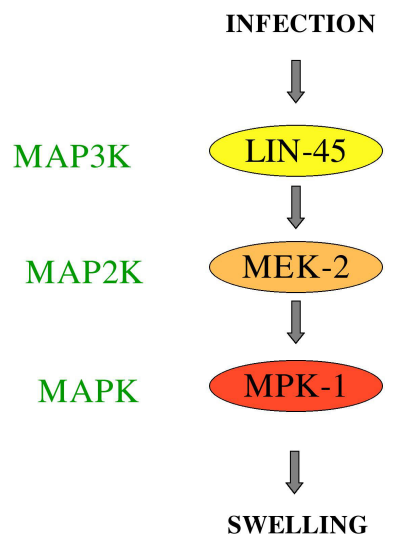

Figure 5. A MAPK cascade involved in the response to infection by M. nematophilum. 


\section{Recognition of infection through stress response}

In common with the other pathways mentioned above, in the case of M. nematophilum it is unclear how the infection is recognised. There still remains the formal possibility that $C$. elegans responds to a stress induced by infection and not to the presence of a pathogen. For example, the P. aeruginosa strain PA01 produces cyanide that paralyses and kills worms (Darby et al., 1999; Gallagher and Manoil, 2001). In common with the response to hypoxia, the response to PA01 involves the oxygen sensor protein EGL-9 that regulates the hypoxia-induced factor HIF-1 (Epstein et al., 2001). egl-9 mutants are resistant to PA01 (Darby et al., 1999), presumably since they have an increased resistance to hypoxia at the cellular level. In a similar manner, it is known that the response to the Cry toxins produced by pathogenic Bacillus thuringiensis involves 2 distinct MAPK pathways (p38 and JNK) and overlap to some degree with the response to abiotic stresses such as heavy metals (Huffman et al., 2004; reviewed in Young and Dillin, 2004) and that the DAF-2/DAF-16 pathway is involved in resistance to heat, UV, hypoxia (Scott et al., 2002), and heavy metals (Barsyte et al., 2001). Further, recent work has identified the MAPK phosphatase VHP 1 as an integrator of the stress response provoked by heavy metals and by infection, revealed the MAP2K MEK-1 to be required for full activation of PMK-1 (Kim et al., 2004; Mizuno et al., 2004), and shown that there is a link between a MAPK pathway and the DAF-2/DAF-16 pathway, as oxidative stress-induced activation of DAF-16 requires the MAP2K SEK-1. Significantly, normal activation of DAF-16 was observed in sek-1 mutants upon heat shock or starvation (Kondo et al., 2005). On the other hand, the nuclear translocation of DAF-16 provoked by heat shock does require jnk-1. Indeed DAF-16 appears to be a direct of JNK-1 phosphorylation (Oh et al., 2005). These results clearly indicate that different pathways intervene in the response to distinct types of stress. These pathways are summarized in Figure 6.

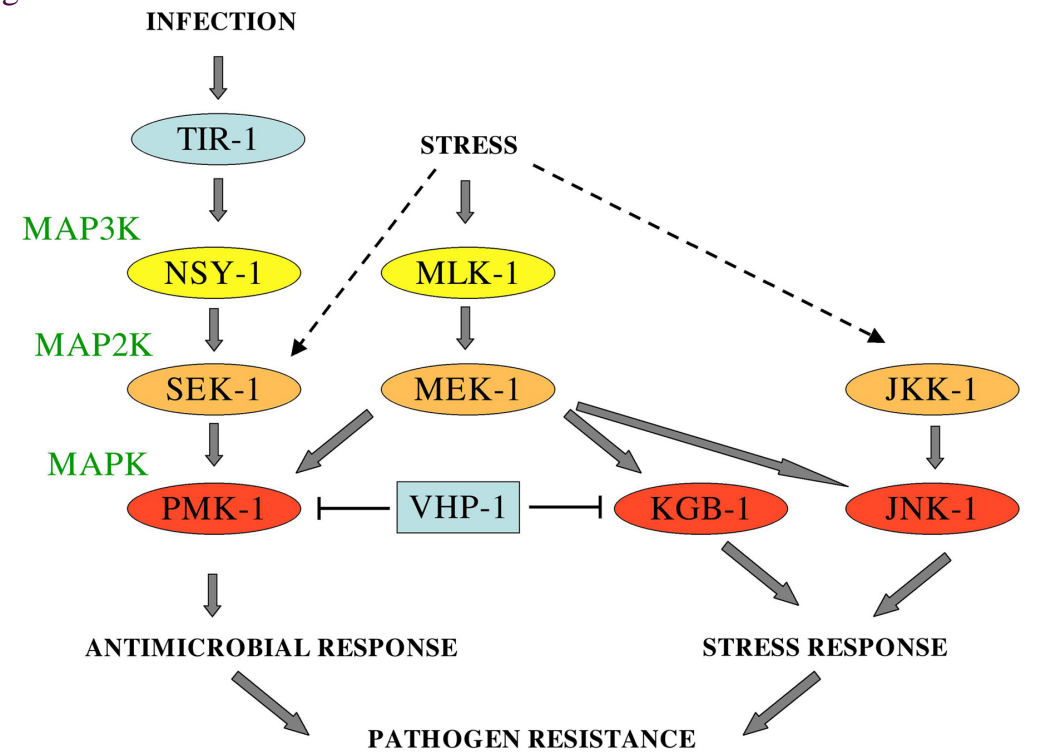

Figure 6. The TIR-1/NSY-1/SEK-1 cassette is part of a broader signaling network. Figure based on published information (Huffman et al., 2004; Kim et al., 2004; Koga et al., 2000; Mizuno et al., 2004; Sakaguchi et al., 2004; Villanueva et al., 2001). The MAP3K presumed to act upstream of SEK-1 and JKK-1 have not been identified. It is noteworthy that there is some uncertainty as to whether JNK-1 really plays a role in the resistance to the stress associated with heavy metal exposure (Mizuno et al., 2004) as originally reported (Villanueva et al., 2001).

Despite these observations, the specificity of the induction of particular genes following infection argues for a more specific recognition and directed response. This is well illustrated for certain members of the $n l p$ family, as individualgenes show distinct patterns of induction despite their similar sequences and close proximity in the genome. For example, $n l p-29$ and $n l p-31$ lie within a cluster of $6 n l p$ genes (Figure 7) but only pnlp-29::gfp shows an intense induction in the peri-vulval cells upon D. coniospora infection (Couillault et al., 2004). Further, different natural isolates of $C$. elegans can show very variable responses to infection, even when taken from the same geographic location (Schulenburg and Ewbank, 2004).

\section{Perspectives}

The study of innate immunity in C. elegans is in its infancy. Nevertheless, considerable advances have already been made in identifying signal transduction pathways involved in the response to a number of pathogens. Little is know about how different cells communicate during the response to infection. Judging from what has been learnt 
about the regulation of longevity and resistance to oxidative stress (e.g., Fujii et al., 2004), it seems probable that the nervous system will play a signaling or integrative role (reviewed in Millet and Ewbank, 2004). Future work will also undoubtedly lead to an understanding of how pathogens are discriminated, as well as addressing the mechanisms involved in the response to intracellular pathogens, including viruses. RNAi is thought to represent an ancient and conserved antiviral response. Although for the moment no models of natural viral infection of $C$. elegans have been established, results from several laboratories strongly support such an idea (Lu et al., 2005; Wilkins et al., 2005; Schott et al., 2005). Another area ripe for study is the cell biology of the response; there are already tantalizing hints of a coordination via RAB-1 of the signaling pathways that lead to antimicrobial peptide expression and the subsequent secretion of these molecules (Couillault et al., 2004). The fact that parts of "innate immunity" pathways function in other essential cellular processes raises a host of issues, such as the primacy of the different roles, the mechanics of their separation and the evolutionary constraints associated with this molecular parsimony (Schulenburg et al., 2004). Work currently underway in an increasing number of laboratories will help answer these questions, and should aid our understanding of vertebrate immunity.

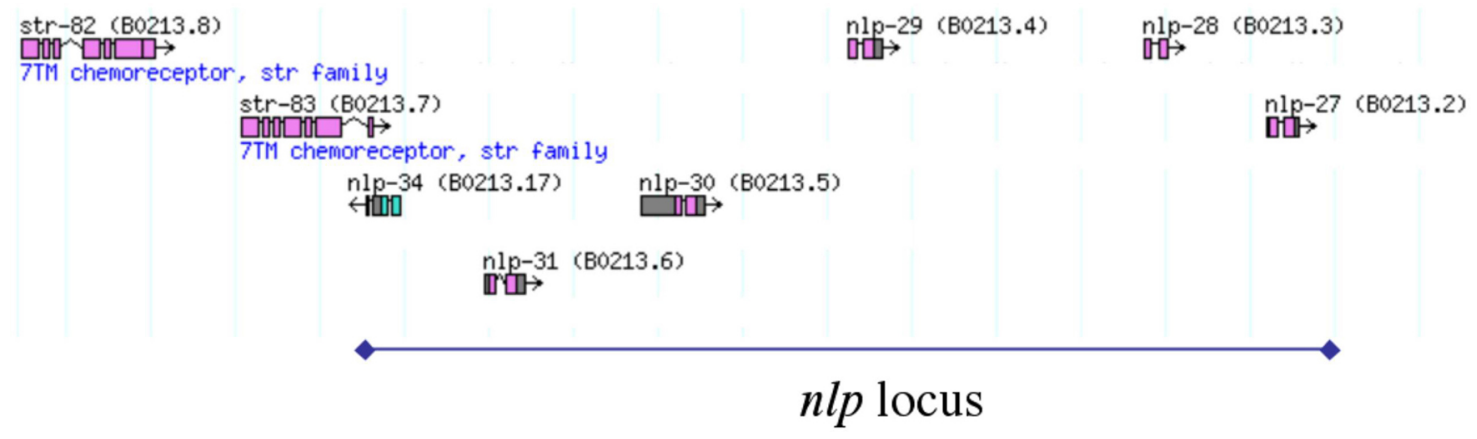

Figure 7. Annotation of part of the sequenced cosmid B0213. Vertical blue lines represent 1 kb intervals. Figure adapted from data in Wormbase.

\section{Acknowledgements}

I thank Léo Kurz, Nathalie Pujol and Hinrich Schulenburg for constructive criticism, former and present members of my group for their contribution to the work described and the different laboratories using C. elegans as a model for the study of host-pathogen interactions for open and stimulating discussion. Work in the lab is supported by INSERM, the CNRS, the French Ministry of Research, the Marseille-Nice genopole ${ }^{\circledR}$ and the European Community (Nemagenetag).

\section{References}

Aballay, A., and Ausubel, F.M. (2001). Programmed cell death mediated by ced-3 and ced-4 protects Caenorhabditis elegans from Salmonella typhimurium-mediated killing. Proc. Natl. Acad. Sci. USA 98, $2735-2739$. Abstract Article

Aballay, A., Drenkard, E., Hilbun, L.R., and Ausubel, F.M. (2003). Caenorhabditis elegans innate immune response triggered by Salmonella enterica requires intact LPS and is mediated by a MAPK signaling pathway. Curr. Biol. 13, 47-52. Abstract Article

Aballay, A., Yorgey, P., and Ausubel, F.M. (2000). Salmonella typhimurium proliferates and establishes a persistent infection in the intestine of Caenorhabditis elegans. Curr. Biol. 10, 1539-1542. Abstract Article

Alegado, R.A., Campbell, M.C., Chen, W.C., Slutz, S.S., and Tan, M.W. (2003). Characterization of mediators of microbial virulence and innate immunity using the Caenorhabditis elegans host-pathogen model. Cell Microbiol. 5, 435-444. Abstract Article

Barsyte, D., Lovejoy, D.A., and Lithgow, G.J. (2001). Longevity and heavy metal resistance in daf-2 and age-1 long-lived mutants of Caenorhabditis elegans. FASEB J. 15, 627-634. Abstract Article 
Chuang, C.F., and Bargmann, C.I. (2005). A Toll-interleukin 1 repeat protein at the synapse specifies asymmetric odorant receptor expression via ASK1 MAPKKK signaling. Genes Dev. 19, 270-281. Abstract Article

Couillault, C., Pujol, N., Reboul, J., Sabatier, L., Guichou, J.F., Kohara, Y., and Ewbank, J.J. (2004). TLR-independent control of innate immunity in Caenorhabditis elegans by the TIR domain adaptor protein TIR-1, an ortholog of human SARM. Nat. Immunol. 5, 488-494. Abstract Article

Darby,C., Cosma,C.L.,Thomas, J.H., and Manoil, C. (1999). Lethal paralysis of Caenorhabditis elegans by Pseudomonas aeruginosa. Proc. Natl. Acad. Sci. USA 96, 15202-15207. Article

Epstein, A.C., Gleadle, J.M., McNeill, L.A., Hewitson, K.S., O'Rourke, J., Mole, D.R., Mukherji, M., Metzen, E., Wilson, M.I., Dhanda, A., et al. (2001). C. elegans EGL-9 and mammalian homologs define a family of dioxygenases that regulate HIF by prolyl hydroxylation. Cell 107, 43-54. Abstract Article

Ewbank, J.J. (2002). Tackling both sides of the host-pathogen equation with Caenorhabditis elegans. Microbes Infect. 4, 247-256. Abstract Article

Fujii, M., Matsumoto, Y., Tanaka, N., Miki, K., Suzuki, T., Ishii, N., and Ayusawa, D. (2004). Mutations in chemosensory cilia cause resistance to paraquat in nematode Caenorhabditis elegans. J. Biol. Chem. 279, 20277-20282. Abstract Article

Gallagher, L.A., and Manoil, C. (2001). Pseudomonas aeruginosa PAO1 kills Caenorhabditis elegans by cyanide poisoning. J. Bacteriol. 183, 6207-6214. Abstract Article

Garsin, D.A., Sifri, C.D., Mylonakis, E., Qin, X., Singh, K.V., Murray, B.E., Calderwood, S.B., and Ausubel, F.M. (2001). A simple model host for identifying Gram-positive virulence factors. Proc. Natl. Acad. Sci. USA 98, 10892-10897. Abstract Article

Garsin, D.A., Villanueva, J.M., Begun, J., Kim, D.H., Sifri, C.D., Calderwood, S.B., Ruvkun, G., and Ausubel, F.M. (2003). Long-lived C. elegans daf-2 mutants are resistant to bacterial pathogens. Science 300, 1921. Abstract Article

Gems, D., and Riddle, D.L. (2000). Genetic, behavioral and environmental determinants of male longevity in Caenorhabditis elegans. Genetics 154, 1597-1610. Abstract

Gravato-Nobre, M.J., Nicholas, H.R., Nijland, R., O'Rourke, D., Whittington, D.E., Yook, K.J., and Hodgkin, J. (2005). Multiple genes affect sensitivity of Caenorhabditis elegans to the bacterial pathogen Microbacterium nematophilum. Genetics 171, 1033-1045. Abstract Article

Herndon, L.A., Schmeissner, P.J., Dudaronek, J.M., Brown, P.A., Listner, K.M., Sakano, Y., Paupard, M.C., Hall, D.H., and Driscoll, M. (2002). Stochastic and genetic factors influence tissue-specific decline in ageing C. elegans. Nature 419, 808-814. Abstract Article

Hodgkin, J., Kuwabara, P.E., and Corneliussen, B. (2000). A novel bacterial pathogen, Microbacterium nematophilum, induces morphological change in the nematode C. elegans. Curr. Biol. 10, 1615-1618. Abstract Article

Huffman, D.L., Abrami, L., Sasik, R., Corbeil, J., van der Goot, F.G., and Aroian, R.V. (2004). Mitogen-activated protein kinase pathways defend against bacterial pore-forming toxins. Proc. Natl. Acad. Sci. USA 101, 10995-11000. Abstract Article

Kanzok, S.M., Hoa, N.T., Bonizzoni, M., Luna, C., Huang, Y., Malacrida, A.R., and Zheng, L. (2004). Origin of Toll-like receptor-mediated innate immunity. J. Mol. Evol. 58, 442-448. Abstract Article

Kato, Y., Aizawa, T., Hoshino, H., Kawano, K., Nitta, K., and Zhang, H. (2002). abf-1 and abf-2, ASABF-type antimicrobial peptide genes in Caenorhabditis elegans. Biochem. J. 361, 221-230. Abstract Article

Kim, D.H., Feinbaum, R., Alloing, G., Emerson, F.E., Garsin, D.A., Inoue, H., Tanaka-Hino, M., Hisamoto, N., Matsumoto, K., Tan, M.W., and Ausubel, F.M. (2002). A conserved p38 MAP kinase pathway in Caenorhabditis elegans innate immunity. Science 297, 623-626. Abstract Article 
Kim, D.H., Liberati, N.T., Mizuno, T., Inoue, H., Hisamoto, N., Matsumoto, K., and Ausubel, F.M. (2004). Integration of Caenorhabditis elegans MAPK pathways mediating immunity and stress resistance by MEK-1 MAPK kinase and VHP-1 MAPK phosphatase. Proc. Natl. Acad. Sci. USA 101, 10990-10994. Abstract Article

Koga, M., Zwaal, R., Guan, K.L., Avery, L., and Ohshima, Y. (2000). A Caenorhabditis elegans MAP kinase kinase, $M E K-1$, is involved in stress responses. EMBO J. 19, 5148-5156. Abstract Article

Kondo, M., Yanase, S., Ishii, T., Hartman, P.S., Matsumoto, K., and Ishii, N. (2005). The p38 signal transduction pathway participates in the oxidative stress-mediated translocation of DAF-16 to Caenorhabditis elegans nuclei. Mech. Ageing Dev. 126, 642-647. Abstract Article

Oh, S.W., Mukhopadhyay, A., Svrzikapa, N., Jiang, F., Davis, R.J., and Tissenbaum, H.A. (2005). JNK regulates lifespan in Caenorhabditis elegans by modulating nuclear translocation of forkhead transcription factor/DAF-16. Proc. Natl. Acad. Sci. USA 102, 4494-4499. Abstract Article

Kurz, C.L., and Ewbank, J.J. (2003). Caenorhabditis elegans: an emerging genetic model for the study of innate immunity. Nat. Rev. Genet. 4, 380-390. Abstract Article

Kurz, C.L., and Tan, M.W. (2004). Regulation of aging and innate immunity in C. elegans. Aging Cell 3, 185-193. Abstract Article

Labrousse, A., Chauvet, S., Couillault, C., Kurz, C.L., and Ewbank, J.J. (2000). Caenorhabditis elegans is a model host for Salmonella typhimurium. Curr. Biol. 10, 1543-1545. Abstract Article

Liang, J., Lints, R., Foehr, M.L., Tokarz, R., Yu, L., Emmons, S.W., Liu, J., and Savage-Dunn, C. (2003). The Caenorhabditis elegans schnurri homolog sma-9 mediates stage- and cell type-specific responses to DBL-1 BMP-related signaling. Development 130, 6453-6464. Abstract Article

Liberati, N.T., Fitzgerald, K.A., Kim, D.H., Feinbaum, R., Golenbock, D.T., and Ausubel, F.M. (2004). Requirement for a conserved Toll/interleukin-1 resistance domain protein in the Caenorhabditis elegans immune response. Proc. Natl. Acad. Sci. USA 101, 6593-6598. Abstract Article

Lu, R., Maduro, M., Li, F., Li, H.W., Broitman-Maduro, G., Li, W.X., and Ding, S.W. (2005). Animal virus replication and RNAi-mediated antiviral silencing in Caenorhabditis elegans. Nature 436, 1040-1043. Abstract Article

Maduzia, L.L., Gumienny, T.L., Zimmerman, C.M., Wang, H., Shetgiri, P., Krishna, S., Roberts, A.F., and Padgett, R.W. (2002). lon-1 regulates Caenorhabditis elegans body size downstream of the dbl-1 TGF [x0392] signaling pathway. Dev. Biol. 246, 418-428. Abstract Article

Mahajan-Miklos, S., Rahme, L.G., and Ausubel, F.M. (2000). Elucidating the molecular mechanisms of bacterial virulence using non- mammalian hosts. Mol. Microbiol. 37, 981-988. Abstract Article

Mahajan-Miklos, S., Tan, M.W., Rahme, L.G., and Ausubel, F.M. (1999). Molecular mechanisms of bacterial virulence elucidated using a Pseudomonas aeruginosa-Caenorhabditis elegans pathogenesis model. Cell 96, 47-56. Article

Mallo, G.V., Kurz, C.L., Couillault, C., Pujol, N., Granjeaud, S., Kohara, Y., and Ewbank, J.J. (2002). Inducible antibacterial defense system in C. elegans. Curr. Biol. 12, 1209-1214. Abstract Article

Millet, A.C., and Ewbank, J.J. (2004). Immunity in Caenorhabditis elegans. Curr. Opin. Immunol. 16, 4-9. Abstract Article

Mizuno, T., Hisamoto, N., Terada, T., Kondo, T., Adachi, M., Nishida, E., Kim, D.H., Ausubel, F.M., and Matsumoto, K. (2004). The Caenorhabditis elegans MAPK phosphatase VHP-1 mediates a novel JNK-like signaling pathway in stress response. EMBO J. 23, 2226-2234. Abstract Article 
Morita, K., Chow, K.L., and Ueno, N. (1999). Regulation of body length and male tail ray pattern formation of Caenorhabditis elegans by a member of TGF- $\beta$ family. Development 126, 1337-1347. Abstract

Morita, K., Flemming, A.J., Sugihara, Y., Mochii, M., Suzuki, Y., Yoshida, S., Wood, W.B., Kohara, Y., Leroi, A.M., and Ueno, N. (2002). A Caenorhabditis elegans TGF- $\beta$,DBL-1, controls the expression of LON-1, a PR-related protein, that regulates polyploidization and body length. EMBO J. 21, 1063-1073. Abstract Article

Murphy, C.T., McCarroll, S.A., Bargmann, C.I., Fraser, A., Kamath, R.S., Ahringer, J., Li, H., and Kenyon, C. (2003). Genes that act downstream of DAF-16 to influence the lifespan of Caenorhabditis elegans. Nature 424, 277-283. Abstract Article

Nicholas, H.R., and Hodgkin, J. (2004). The ERK MAP kinase cascade mediates tail swelling and a protective response to rectal infection in C. elegans. Curr. Biol. 14, 1256-1261. Abstract Article

Pujol, N., and Ewbank, J.J. (2003). Pathogen avoidance using Toll signalling. In Toll Receptors, T. Rich, ed. (Georgetown, TX: Landes Bioscience Press).

Pujol, N., Link, E.M., Liu, L.X., Kurz, L.C., Alloing, G., Tan, M.W., Ray, K.P., Solari, R., Johnson, C.D., and Ewbank, J.J. (2001). A reverse genetic analysis of components of the Toll signalling pathway in Caenorhabditis elegans. Curr. Biol. 11, 809-821. Abstract Article

Sagasti, A., Hisamoto, N., Hyodo, J., Tanaka-Hino, M., Matsumoto, K., and Bargmann, C.I. (2001). The CaMKII UNC-43 activates the MAPKKK NSY-1 to execute a lateral signaling decision required for asymmetric olfactory neuron fates. Cell 105, 221-232. Abstract Article

Sakaguchi, A., Matsumoto, K., and Hisamoto, N. (2004). Roles of MAP kinase cascades in Caenorhabditis elegans. J. Biochem. (Tokyo) 136, 7-11. Abstract Article

Schulenburg, H., and Ewbank, J.J. (2004). Diversity and specificity in the interaction between Caenorhabditis elegans and the pathogen Serratia marcescens. BMC Evol. Biol. 4, 49. Abstract Article

Schulenburg, H., Kurz, C.L., and Ewbank, J.J. (2004). Evolution of the innate immune system: the worm perspective. Immunol. Rev. 198, 36-58. Abstract Article

Scott, B.A., Avidan, M.S., and Crowder, C.M. (2002). Regulation of hypoxic death in C. elegans by the insulin/IGF receptor homolog DAF-2. Science 296, 2388-2391. Abstract Article

Schott, D.H., Cureton, D.K., Whelan, S.P., and Hunter, C.P. (2005). An antiviral role for the RNA interference machinery in Caenorhabditis elegans. Proc. Natl. Acad. Sci. USA 102, 18420-18424. Abstract Article

Seet, B.T., and Pawson, T. (2004). MAPK signaling: Sho business. Curr. Biol. 14, R708-R710. Abstract Article

Tan, M.W. (2001). Genetic and genomic dissection of host-pathogen interactions using a P. aeruginosa-C. elegans pathogenesis model. Pediatr. Pulmonol. 32, 96-97.

Tan, M.W., Mahajan-Miklos, S., and Ausubel, F.M. (1999a). Killing of Caenorhabditis elegans by Pseudomonas aeruginosa used to model mammalian bacterial pathogenesis. Proc. Natl. Acad. Sci. USA 96, 715-720. Article

Tan, M.W., Rahme, L.G., Sternberg, J.A., Tompkins, R.G., and Ausubel, F.M. (1999b). Pseudomonas aeruginosa killing of Caenorhabditis elegans used to identify P. aeruginosa virulence factors. Proc. Natl. Acad. Sci. USA 96, 2408-2413. Article

Tanaka-Hino, M., Sagasti, A., Hisamoto, N., Kawasaki, M., Nakano, S., Ninomiya-Tsuji, J., Bargmann, C.I., and Matsumoto, K. (2002). SEK-1 MAPKK mediates $\mathrm{Ca}^{+}$signaling to determine neuronal asymmetric development in Caenorhabditis elegans. EMBO Rep. 3, 56-62. Abstract Article

Villanueva, A., Lozano, J., Morales, A., Lin, X., Deng, X., Hengartner, M.O., and Kolesnick, R.N. (2001). jkk-1 and mek-1 regulate body movement coordination and response to heavy metals through jnk-1 in Caenorhabditis elegans. EMBO J. 20, 5114-5128. Abstract Article 
Wilkins, C., Dishongh, R., Moore, S.C., Whitt, M.A., Chow, M., Machaca, K. (2005). RNA interference is an antiviral defence mechanism in Caenorhabditis elegans. Nature 436, 1044-1047. Abstract Article

Young, J.A., and Dillin, A. (2004). MAPping innate immunity. Proc. Natl. Acad. Sci. USA 101, 12781-12782. Abstract Article

All WormBook content, except where otherwise noted, is licensed under a Creative 